Research Article

\title{
Relationship between post-op central corneal thickness and steroid-induced ocular hypertension following myopic photorefractive keratectomy
}

\begin{abstract}
Purpose: Purpose of this study is to determine the relationship between Post-op central corneal thickness and steroid-induced ocular hypertension following myopic photorefractive keratectomy.

Methods: This longitudinal analytical study was carried on 50 patients, both gender and age ranging from 20-40 years who had undergone myopic PRK refractive surgery. The data collected from The Department of Ophthalmology in Madina Teaching Hospital Faisalabad in the duration of 11 months from July 2019 to May 2020. IOP measured by Goldmann applanation tonometer. Recorded IOP greater than $21 \mathrm{mmHg}$ was considered as Ocular hypertension (OHT). The post-Op follow-up was conducted 1 day, 1 week, 2-3 weeks, and 4-6 weeks. The CCT and IOP was evaluated carefully. Data was noted on Performa. Data was analyzed by using Descriptive and Repeated Measures ANOVA by using the latest version 21 of SPSS.

Results: Out of 50 patients' $46 \%$ were males and $54 \%$ were females. The mean score of CCT pre \& post operatively for right eye and left eye was $529.28 \pm 34.74 \& 462.56 \pm 40.20$ and $529.18 \pm 37.2,462.44 \pm 42.60$ respectively. Statistically significant effect was seen on post-op central corneal thickness and steroid induced ocular hypertension, according to the results of repeated measure ANOVA. Thinner corneas were more susceptible to steroids induced OHT. In various post-op central corneal thickness, it was observed that patients with thinner corneal thickness ranges $413 \pm 4.24$ to $449.33 \pm 48.29$ in right eye and $455.8 \pm 56.42$ to $456.83 \pm 50.52$ are more affected by topical steroids used. Males were more affected than females due to decreased central corneal thickness. Elderly patients were more susceptible to steroid-induced ocular hypertension post-operatively.

Conclusion. Post-op steroid induced ocular hypertension is affecting by central corneal thickness. Male and elderly individual are more susceptible to steroid-induced ocular hypertension following myopic PRK. Post-Op use of topical steroids cause ocular hypertension in thinner corneas.
\end{abstract}

Keywords: CCT, PRK, ocular hypertension, steroids
Volume I0 Issue 5 - 2020

\author{
Hina Manzoor,' Asma Batool, ${ }^{2}$ Saba Akram, ${ }^{2}$ \\ Iqra Khalil, ${ }^{3}$ Ammara Affi' \\ 'Lecturer, University of Lahore, Pakisthan \\ ${ }^{2}$ Senior Lecturer, University of Lahore, Pakisthan \\ ${ }^{3}$ Optometrist at Aziz Fatima hospital, Pakisthan
}

\begin{abstract}
Correspondence: Hina Manzoor, Lecturer, University of Lahore, MCB 9/1008 near nishat cinema mohallah eid gah Chakwal, Pakisthan, Email HYPERLINK"mailto:hinamanzoor05@gmail. com”hinamanzoor05@gmail.com
\end{abstract}

Received: October 05, 2020 | Published: November 23, 2020

\section{Introduction}

Photorefractive keratectomy is a laser procedure widely used for the correction of myopia and astigmatism. In this procedure we modify the anterior corneal surface in order to flatten the curvature of the cornea. For the treatment of myopia PRK believed to be effective and safe. Refractive PRK is a procedure of choice for individuals who are not allowed for LASIK due to thinner cornea, subtle topographical irregularities and epithelium basement membrane diseases. ${ }^{1,2}$ In PRK Excimer laser used to reshape the front surface of the cornea. Post-PRK complications involve regression in myopia and hazy cornea, these may lead to multiple attempts for required corrections. In Certain studies, it is reported that the use of topical steroids can resolve sub epithelial haze and inhibit myopic regression. One of the side effects of steroid administration is the increase of intraocular pressure that result in ocular hypertension, steroid responders and glaucoma patients are more susceptible to this. Goldmann applanation tonometer is used to measure intraocular pressure; however, there are various errors effecting the measurements of GAT such as Central corneal thickness. Many studies have shown that thinning and thickening of cornea may results in pseudo decrease and increase in IOP readings respectively. OHTS studies reported that CCT causes changes in IOP readings and progression of ocular hypertension leads to primary open-angle glaucoma which is at greater risk due to low CCT. Therefore, factors affecting CCT should be evaluated properly. Ehlers have shown that $\mathrm{CCT}$ is responsible for correcting IOP in normal corneas, in which 5 $\mathrm{mm} \mathrm{Hg}$ for every $70-\mu \mathrm{m}$ difference in CCT from $545 \mu \mathrm{m}$ is adjusted. A practical approach should be used to measure the IOP pre and post operatively in patients who are going through refractive surgery and the difference of these measurements is used as the correction factor for the patients of PRK. ${ }^{3,4}$ It is recommended that the use of corticosteroid activates the production of molecular myocillin gene. Corticosteroids administration elevates IOP within 2-6 weeks typically. They cause the elevation of the IOP by decreasing the facility of aqueous outflow at the position of the trabecular meshwork. ${ }^{5}$ Reduced aqueous humor outflow occurs after the use of topical, periocular or oral glucocorticoid medications in patient's sensitive to steroids which leads higher IOP. Traditional IOP-lowering therapy includes topical oral medications, laser trabeculoplasty and incisional surgery. Post PRK antibiotics topical anesthetics and corticosteroids are prescribed routinely. So the greater risk of steroid-induced ocular hypertension invloves Higher Central Corneal Thickness, Lower K Value, Male Sex, Corneal Haze, High Myopia, Form of Steroid used and Span of Therapy., 


\section{Material and methodology}

This longitudinal analytical study was carried on 50 subjects through non-probability sampling technique, both gender and age ranging from 20 to 40 years who had underwent myopic photorefractive keratectomy was taken in the study. There were 23 males and 27 females in the study. The study was conducted at Madinah Teaching Hospital (MTH) Faisalabad in duration of 11months from JULY 2019 to MAY 2020. Data was collected after taking extensive history and consent. Visual acuity was tested by using Snellen chart and IOP measured by Goldmann applanation tonometer and central corneal thickness measured by pentacam scan. According to CCT (measured by pentacam scan) the IOP readings were corrected. For the study, corrected IOP greater than $21 \mathrm{mmHg}$ was considered as Ocular hypertension (OHT). The follow-up examination was scheduled 1 day, 1 week, 2-3 weeks, and 4-6 weeks postoperatively, furthermore the IOP, CCT, and corneal curvatures measurements were recorded. Data was noted on Performa. Data was analyzed by using Descriptive and Repeated Measures ANOVA by using the latest version 21 of SPSS.

\section{Results}

Out of 50 participants' $46 \%$ were males and $54 \%$ were females The mean score of CCT pre \& post operatively for right eye and left eye was $529.28 \pm 34.74,462.56 \pm 40.20$ and $529.18 \pm 37.2,462.44 \pm 42.60$ respectively. In Right eye, frequency of Post-op steroid-induced OHT was $4 \%, 8 \%, 10 \%$, and $4 \%$ at 24 hours, 1 week, $3-4$ weeks, and $4-6$ weeks. In left eye, frequency was $4 \%, 10 \%, 12 \%$, and $4 \%$ at follow-ups (Graph 1). In which maximum post-operative rise intraocular pressure was $28 \mathrm{mmHg}$ in 3-4 weeks in both eyes. Significant correlation was observed between Post-op central corneal thickness and raised IOP in both eyes. A result of repeated measures ANOVA test with-in-subjects in right and left eye (post-op CCT). The significance of Mauchly's Test of Sphericity table is showing that the significance is 0.360 (right eye) 0.375 (left eye) and Greenhouse-Geisser is 0.353 (right eye) 0.366 (left eye). Value of Mauchly's W is 0.015 and 0.016 for right and left eyes. According to the results of repeated measure ANOVA on intraocular pressure with-in-subjects it is seen that there is statistically significant effect of the post-op central corneal thickness on steroid induced ocular hypertension. Overall, result shown that thinner corneas are more susceptible to steroids induced OHT. In various post-op central corneal thickness, it was observed that patients with thinner corneal thickness ranges $413 \pm 4.24$ to $449.33 \pm 48.29$ in right eye and $455.8 \pm 56.42$ to $456.83 \pm 50.52$ are more affected by topical steroids used (shown in Tables 1\&2). Males were more affected than females due to decreased central corneal thickness. Elderly patients were more susceptible to steroid-induced ocular hypertension postoperatively (shown in Table 3).

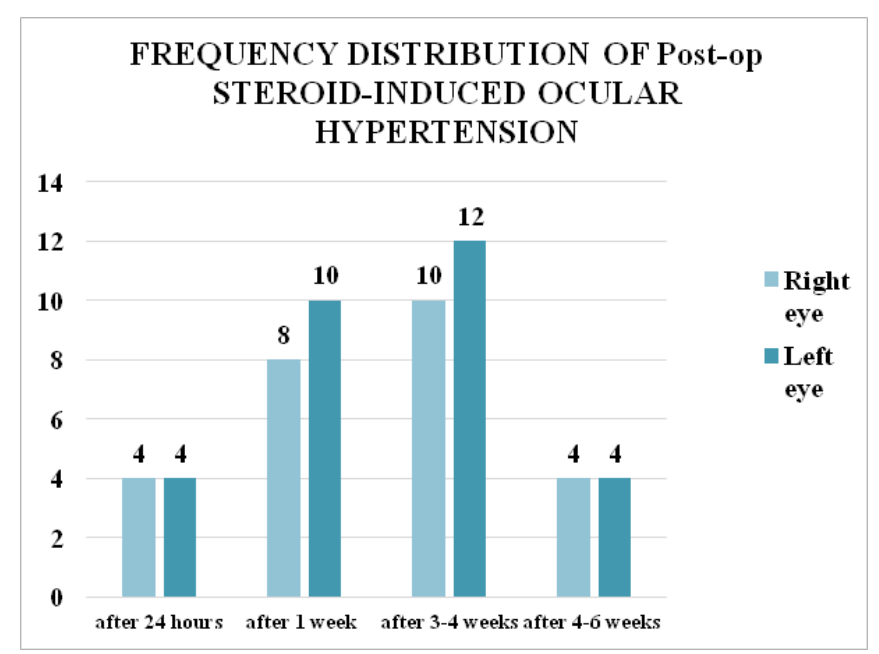

Graph I Frequency distribution of Post-op steroid-induced ocular hypertension.

Table I Comparison of CCT in participants with and without Ocular hypertension (right eye)

\begin{tabular}{lll}
\hline \multirow{2}{*}{ Comparison of CCT in participants with and without Ocular hypertension (right eye) } & \multicolumn{3}{l}{ Intraocular Hypertension (OHT) } \\
\cline { 2 - 4 } & Yes & No \\
\hline After 24 hours & $413 \pm 4.24$ & $464.63 \pm 39.69$ \\
After I week & $439.75 \pm 59$ & $464.54 \pm 38.44$ \\
3-4 weeks & $449.33 \pm 48.29$ & $464.36 \pm 39.28$ \\
4-6 weeks & $512 \pm 22.63$ & $460.5 \pm 39.57$ \\
\hline
\end{tabular}

Table 2 Comparison of CCT in participants with and without Ocular hypertension (left eye)

Comparison of CCT in participants with and without Ocular hypertension (left eye)

After 24 hours

After I week

3-4 weeks

4-6 weeks
Ocular Hypertension (OHT)

$\begin{array}{ll}\text { Yes } & \text { No } \\ 455.8 \pm 56.42 & 463.18 \pm 41.55 \\ 462.71 \pm 48.67 & 462.4 \pm 42.18 \\ 456.83 \pm 50.52 & 463.2 \pm 42.03 \\ 486.67 \pm 51.98 & 460.89 \pm 42.13\end{array}$

Table 3 Age based distribution with/without post-op steroid induced-ocular hypertension

\begin{tabular}{lllll}
\hline & & N & Minimum & Maximum \\
\hline Right & With OHT & 5 & 22 & 28 \\
Eye & Without OHT & 45 & 20 & 25 \\
Left & With OHT & 6 & 23 & 31 \\
Eye & Without OHT & 44 & 20 & 25 \\
\hline
\end{tabular}


Description graph 1: Frequency based distribution of Post-op steroid-induced ocular hypertension shows that Post-op steroidinduced ocular hypertension was found $4 \%, 8 \%, 10 \%$, and $4 \%$ at 24 hours, 1 week, 3-4 weeks and 4-6 weeks in right eye respectively. Post-op steroid-induced ocular hypertension was found $4 \%, 10 \%$, $12 \%$, and $4 \%$ in left eye respectively.

\section{Table I}

Description: The mean score of CCT (right eye) of PRK found $413 \pm 4.24,439.75 \pm 59,449.33 \pm 48.29$ and $512 \pm 22.63$ after 24 hours, 1 week,3-4 weeks,4-6 weeks of PRK in participants with and without $464.63 \pm 39.69,464.54 \pm 38.44,464.36 \pm 39.28$ and $460.5 \pm 39.57$ post-op steroid induced ocular hypertension respectively.

\section{Table 2}

Description: The mean score of CCT (left eye) of PRK found $455.8 \pm 56.42,462.71 \pm 48.67,456.83 \pm 50.52$ and $486.67 \pm 51.98$ after 24 hours, 1 week,3-4 weeks,4-6 weeks of PRK in participants with and without $463.18 \pm 41.55,462.4 \pm 42.18,463.2 \pm 42.03$ and $460.89 \pm 42.13$ post-op steroid induced ocular hypertension respectively. The thinner corneas are more susceptible to steroids induced OHT as compare to thicker corneas.

\section{Table 3}

Age based description: result show that steroid induced ocular hypertension is more common with increasing age. Between 22-31 years old were steroid-induced ocular hypertension and 20-25 without steroid-induced ocular hypertension.

\section{Discussion}

The main objective of this study is to find relationship between corneal thickness and raised IOP induced by steroids after photorefractive keratectomy in myopic individuals. Those who underwent myopic photorefractive keratectomy (PRK) participated in this study. Our study includes 50 patients of which $46 \%$ are males and $54 \%$ are females. The mean age of the participants is $26.88 \pm 4.38$. The mean value of Central corneal thickness (CCT) for right eye before surgery is $529.28 \pm 34.74$ and for left eye is $529.18 \pm 37.2$. After surgery the mean score is $462.56 \pm 40.20$ in Right and $462.44 \pm 42.60$ in left eye. This study shows highly significant difference in central corneal thickness before and after photorefractive keratectomy (PRK), there is reduction in thickness of central cornea with mean score of $462.56 \pm 40.20$ and $462.44 \pm 42.20$ in right and left eye respectively. This study also provides the information that thinner corneas need more pre-operative care than thick or normal corneas (540 and 550). Naderi et al. in a recent study has given indication of the efficacy and safety of surface ablation in subjects with $<500-\mu m$-thick corneas, hence providing new measures to the surgical treatment of these patients. Conversely, Kanellopoulous et al., ${ }^{7} 2016$ compared intraocular pressure changes between two groups (keratoconic versus normal eyes) following topical dexamethasone administration for 1 month. He stated that corneal thickness decreasing alone may not describe the difference between IOP elevation after surgery. but he also concludes that keratoconic is more prone to ocular hypertension due to thin corneal thickness as compare to the normal eye. Similarly, T Yamaguchi, 2008 study conclude that there is chance of steroidinduced glaucomic complication in PRK surgery, whereby the exact IOP may be underestimated due to decrease in corneal thickness. Corneas are especially thin in the Japanese population, necessitating caution for IOP assessment during steroid use.
This study describes the impact of post-operative (PRK) steroids on intraocular pressure (IOP), as steroids enhance the tension in eyes. The mean values for Intraocular pressure (IOP) in right eye and left eye is not statistically significant because our sample size is small. The first report on the intraocular pressure-increasing outcomes of adrenocorticotropic hormone and cortisone was introduced by McLean. Armaly in 1960s characterized steroid-induced glaucoma. These researchers reported that $34 \%$ to $42 \%$ of normal subjects had an increase in intraocular pressure from a starting point of 6 to $15 \mathrm{mmHg}$ to end value of 20 to $31 \mathrm{mmHg}$ after dexamethasone or betamethasone administered 3-4 times a day for four weeks. Numerous studies have examined the result of PRK on the intra-ocular variation with the applanation tonometer. Faucher and his colleagues in the $1997^{10}$ described a mean decrease of $2.4 \mathrm{mmHg}$, Chatterjee found a decrease of $3.1 \mathrm{mmHg}$, Schipper, and his co-worker found a decrease in intraocular pressure of 2 to $3 \mathrm{mmHg}$ after PRK. Conversely, Kohlhaas and his team described an increase of $0.1 \mathrm{mmHg}$. Our study found that after Trans-PRK surgery IOP revealed a rising pattern as compared to baseline IOP. Four-six weeks postoperatively, the mean adjusted according to CCT IOP was $17 \pm 3.64 \mathrm{mmHg}$, which was $0.23 \pm 1.08$ $\mathrm{mmHg}$ greater than the mean baseline IOP value. Postoperatively, the adjusted IOP decreased, but can't be reached to baseline value. ${ }^{11-13}$

\section{Conclusion}

This study was conducted to find out the relationship between postop central corneal thickness and steroid-induced ocular hypertension following myopic photorefractive keratectomy. It was also found out which post-op central corneal thickness, gender, and age are more affected by steroids used after Trans-PRK procedure. This study concluded the positive correlation between post-Op CCT and steroidinduced OHT. Topical steroids cause ocular hypertension in thinner CCT following myopic Trans-PRK. It was also concluded that male and grown-up age was more susceptible to steroid-induced ocular hypertension postoperatively. The early finding and close follow-up especially in thinner corneas are advised to avoid steroid induced OHT.

\section{Acknowledgments}

None.

\section{Funding}

Self funding.

\section{Conflicts of interest}

Authors don't have any conflict of interest and financial disclosure.

\section{References}

1. Naderi M, Ghadamgahi S, Jadidi K. Photorefractive keratectomy (PRK) is safe and effective for patients with myopia and thin corneas. Medical hypothesis, discovery and innovation in ophthalmology. 2016;5(2):58.

2. Hamid Khakshoor, Fatemeh Razavi, Alireza Eslampour, et al Photorefractive keratectomy in mild to moderate keratoconus: outcomes in over 40-year-old patients. Indian journal of ophthalmology. 2015;63(2):157.

3. Kaweh Mansouri, Robert N Weinreb. Ambulatory 24-h intraocular pressure monitoring in the management of glaucoma. Current opinion in ophthalmology. 2015;26(3):214-220.

4. Ted S Acott, Mary J Kelley, Kate E Keller, et al. Intraocular pressure homeostasis: maintaining balance in ahigh-pressure environment.Journal of Ocular pharmacology and therapeutics. 2014;30(2-3):94-101. 
5. Tanuj Dada, Soman Nair, Dhawan M. Steroid-induced Glaucoma Current glaucoma practice. 2009;3:33-38.

6. Yumna Busool, Michael Mimouni, Igor Vainer, et al. Risk factors predicting steroid-induced ocular hypertension after photorefractive keratectomy. J Cataract Refract Surg. 2017;43:389-393.

7. Kanellopoulos AJ, Cruz EM, Ang RET, et al. Higher incidence of steroid-induced ocular hypertension in keratoconus. Eye and Vision. 2016;3(1):4.

8. Yamaguchi T, Murat D, Negishi K, et al. Diagnosis of steroid-induced glaucoma after photorefractive keratectomy. Journal of Refractive Surgery. 2008;24(4):413-415.

9. McLean JM, Woods AC. Clinical and experimental observation on the use of ACTH and cortisone in ocular inflammatory disease. 1950.
10. Faucher A, Grégoire J, Blondeau P. Accuracy of Goldmann tonometry after refractive surgery. Journal of Cataract \& Refractive Surgery. 1997;23(6):832-838.

11. Schipper I, Senn P, Thomann U, et al. Intraocular pressure after excimer laser photorefractive keratectomy for myopia. Journal of refractive surgery. 1995;11(5):366-406.

12. Chatterjee A, Shah S, Bessant DA, et al. Reduction in intraocular pressure after excimer laser photorefractive keratectomy: correlation with pretreatment myopia. Ophthalmology. 1997;104(3):355-359.

13. Kohlhaas M, Boehm AG, Spoerl E, et al. Effect of central corneal thickness, corneal curvature, and axial length on applanation tonometry. Archives of ophthalmology. 2006;124(4):471-476. 